\title{
EDUCACIÓN SUPERIOR EN ALTERNANCIA TRANSFRONTERIZA ENTRE FRANCIA Y ESPAÑA
}

\author{
(HIGHER EDUCATION IN CROSS-BORDER ALTERNATION BETWEEN \\ FRANCE AND SPAIN)
}

Elisenda Tarrats-Pons

Núria Arimany-Serrat

Universitat de Vic-Universitat Central de Catalunya

Saioa Arando-Lasagabaster

Mariangélica Martínez-González

Universidad de Mondragón

Laetitia Mathieu

IAE Pau-Bayonne Ecole Universitaire de Management

Marion Samonneau

Veronique Pilnieri

ESTIA-Ecole Superieure des Technologies Industrielles Avancées, Francia

DOI: $10.5944 / e d u c X X 1.17786$

\section{Cómo referenciar este artículo/How to reference this article:}

Tarrats-Pons, E.; Arimany-Serrat, N.; Arando-Lasagabaster, S.; Martínez-González, M.; Mathieu, L.; Samonneau, M. y Pilnieri, V. (2018). Educación superior en alternancia transfronteriza entre Francia y España. Educación XX1, 21(2), 275-300, doi: 10.5944/educXX1.17786

Tarrats-Pons, E.; Arimany-Serrat, N.; Arando-Lasagabaster, S.; Martínez-González, M.; Mathieu, L.; Samonneau, M. \& Pilnieri, V. (2018). Educación superior en alternancia transfronteriza entre Francia y España. [Higher education in cross-border alternation between france and spain]. Educación XX1, 21(2), 275-300, doi: 10.5944/educXX1.17786

\section{RESUMEN}

En este estudio se pretende evidenciar que la educación en alternancia estimula la creación de empleo e incentiva la innovación acorde a la revolución tecnológica actual, en sintonía con las necesidades de nuestro tiempo en el territorio transfronterizo. Por este motivo se analizan 4 experiencias en la zona transfronteriza basadas en educación superior en alternancia a ambos lados de los Pirineos, como experiencias en sintonía 
con el modelo de la Triple Hélice. Las instituciones de educación superior implicadas en las cuatro experiencias son: Universidad de Mondragón, Universidad de Vic-Universidad Central de Catalunya (en España), la École Superieure des Technologies Industrielles Avancées (ESTIA) y la Escuela Universitaria de Management de la Université de Pau (en Francia). Nuestros hallazgos sugieren los elementos que debería incorporar un modelo de educación en alternancia transfronteriza, considerando los aspectos comunes y discrepantes del análisis de las 4 experiencias, y la pertinencia de utilizar esta innovación educativa para mejorar la empleabilidad y promover la incorporación de los alumnos al mercado laboral junto con el oportuno desarrollo regional, económico y social de las zonas analizadas.

\section{PALABRAS CLAVE}

Innovación Educativa; Educación Superior; Educación Transfronteriza; Formación en Alternancia; Modelo de la Triple Hélice.

\section{ABSTRACT}

In this study, our aim is to prove that education alternatively stimulates the creation of employment and encourages innovation according to the current technological revolution, according to the current necessities in the cross-border territory. For this reason, four experiences are analyzed in this cross-border area, based on higher education in alternation to both sides of the Pyrenees as experiences in tune with the triple helix model. The institutions of higher education involved are Universidad de Mondragon, Universidad de Vic-Universidad Central de Catalunya (Spain), la Escuela Universitaria de management de la Université de Pau (France) and École Superieure des Technologies Industrielles Avancées (ESTIA). Our findings suggest the elements that should have an alternating cross-border education model considering the common and diverging issues of the analysis of the four experiences and the relevance of using this educative innovation to improve employability and promote the market entry of the students along with the regional, social and economic development of the areas under study.

\section{KEYWORDS}

Educational Innovation; Higher Education; Crossborder Education; Alternating Training; Triple Helix Model. 
E. TARRATS-PONS, N. ARIMANY-SERRAT, S. ARANDO-LASAGABASTER, M. MARTÍNEZ-GONZÁLEZ,

\section{INTRODUCCIÓN}

Las economías y las sociedades están en constante cambio: la globalización, el progreso tecnológico y los cambios sociales han marcado el devenir de las últimas décadas. Europa, en su afán de mantener su competitividad en el contexto mundial ha apostado por un crecimiento económico sostenible, inclusivo e integrador. Si bien para ello cuenta con una población cada vez más formada, en algunos países es latente el gap existente entre la oferta y la demanda del mercado laboral, lo que se traduce en tasas de desempleo superiores al $15 \%$ en general y al $40 \%$ en el colectivo de los jóvenes (Eurostat, 2014. Ver tabla 1).

Asimismo, el impacto que está teniendo la crisis económica en la estructura productiva y social de los países europeos se ha trasladado a las instituciones universitarias. El Plan Bolonia está siendo el mecanismo que muchos gobiernos europeos han promovido para tratar de articular el espacio educativo con el mercado productivo a través de la capacitación laboral de los graduados, siendo la educación basada en competencias la posible respuesta a la necesidad de vincular ambos sectores (Abdala, 2004; De Miguel, 2006).

Paradójicamente, nos encontramos en un contexto en el que la sociedad tiene un nivel de educación nunca alcanzado con anterioridad, pero con carencia de personas con competencias críticas que son las que demandan las organizaciones (Mourshed et al. 2016). De hecho, tal y como sugiere Eurostat el porcentaje de la población que cuenta con estudios superiores alcanza el $28,5 \%$ en la Euro área, e incluso asciende al $47 \%$ en algunas regiones europeas como es el caso del País Vasco.

Tabla 1

Indicadores de desempleo y nivel de estudios en la euro área y regiones transfronterizas francoespañolas

\begin{tabular}{lcrcc}
\hline & Euro-28 & Aquitania & Cataluña & $\begin{array}{c}\text { País } \\
\text { Vasco }\end{array}$ \\
\hline Población con estudios superiores (25 a 64 años) & $28,5 \% 1$ & $32,7 \%$ & $37,0 \%$ & $47,0 \%$ \\
Tasa de desempleo \% & $10,2 \%$ & $9,0 \%$ & $20,3 \%$ & $16,3 \%$ \\
Tasa de desempleo juvenil & $22,2 \%$ & $23,8 \%$ & $47,1 \%$ & $45,0 \%$ \\
\hline
\end{tabular}

Fuente: Eurostat, 2014.

Es más, este gap puede que se amplíe debido a los constantes cambios que se auguran para el futuro próximo en los entornos laborales y que tendrán evidentes repercusiones sobre el trabajo de las personas y, 
por tanto, sobre las cualificaciones requeridas para el acceso al mercado laboral (Echaburi, 2003). Una adecuada oferta formativa adaptada a las necesidades de las organizaciones y que facilite el acceso de las personas al mercado laboral y su continuidad contribuirían significativamente a la reducción de la elevada tasa de desempleo en general especialmente para el colectivo juvenil en particular (tabla 1), además de favorecer el crecimiento sostenible, inclusivo e integrador que persigue Europa.

Actualmente el mercado laboral demanda profesionales y trabajadores altamente competitivos con competencias específicas, más allá de sus cualidades intelectuales. Según un estudio realizado por el World Economic Forum en el año 2020 las organizaciones demandarán a profesionales que cuenten con las siguientes competencias: gestión de la complejidad, pensamiento crítico, creatividad, gestión de personas, capacidad de coordinación, inteligencia emocional, capacidad de opinar y decidir, orientación al servicio, capacidad de negociación y flexibilidad cognitiva (Future of Jobs report. World Economic Forum, 2016). En este sentido se está requiriendo a las universidades, acorde a la reforma europea del sistema universitario, que su formación esté orientada a la creación de habilidades y competencias sociales y profesionales que permitan al individuo hacer frente a los actuales desafíos laborales (Luzón Trujillo et al., 2009). El sistema educativo debe formar a profesionales con los conocimientos y competencias que requieren las organizaciones. No obstante, surge la pregunta de si las organizaciones educativas están preparadas para formar a los futuros profesionales en estas competencias y si el entorno educativo es el idóneo para la adquisición de estas competencias.

Esta situación implica una redefinición de los procesos de enseñanza y aprendizaje basada en conocimientos aplicados, útiles social y económicamente (Barnett, 1993). En este contexto, la formación en alternancia se erige como una alternativa que aproxima la experiencia de aprendizaje en el contexto universitario a la realidad del entorno socioeconómico (Comisión Europea, 2012). Bajo este modelo formativo los estudiantes pasan a jugar un rol más activo en su formación, relacionando teoría y práctica desde una visión más holística e integral, para llegar a alcanzar un aprendizaje abierto, flexible, permanente y realista (Huerta et al., 2000). Se trata de un proceso educativo inductivo que otorga valor al saber pragmático y junto a la adquisición de conocimientos cognitivos propios de la didáctica deductiva (lógica aplicacionista), permite la construcción y el desarrollo del pensamiento adaptable entre universidad y entorno laboral (Coiduras, 2013). Esta transformación del sistema educativo debe estar avalada por las instituciones públicas, tal y como sugiere el modelo de la Triple Hélice, que favorece la interacción de universidades/alumnos, empresas y gobiernos y se puede utilizar como estrategia operacional para el desarrollo regional de zonas que desean implementar un ambiente propicio 
para la innovación, el desarrollo económico, la optimización de recursos y el bienestar social (Inzelt, 2004; Marques et al. 2006; Cooke \& Leydesdorff 2006; Saad et al. 2008; Brännback et al. 2008; Dezhina, 2014; Doh \& Kim, 2014; Balzer \& Askonas 2016).

Actualmente, aunque sean escasas, existen prácticas basadas en el modelo de la Triple Hélice, en el territorio transfronterizo a ambos lados de los Pirineos que favorecen la interacción entre universidad/alumnos-empresas y gobiernos. Este artículo tiene por objetivo describir cuatro experiencias basadas en educación superior en alternancia a ambos lados de los pirineos, como experiencias en sintonía con el modelo de la Triple Hélice (en adelante $\mathrm{TH})$, para identificar los elementos que debería incluir un modelo transversal transfronterizo que favorezca el desarrollo y la empleabilidad de este territorio. Las instituciones de educación superior implicadas en las cuatro experiencias son: Universidad de Mondragón, Universidad de Vic-Universidad Central de Catalunya (en España), École Superieure des Technologies Industrielles Avancées (ESTIA) y Université de Pau (en Francia).

En este estudio exploratorio identificamos prácticas educativas de alternancia e innovación en las zonas analizadas susceptibles de ser aplicadas con transversalidad en toda la zona de cooperación transfronteriza; con voluntad de crear ecosistemas de apoyo a la $\mathrm{I}+\mathrm{D}+\mathrm{i}$ en la zona objeto de estudio.

Después de la oportuna revisión de la literatura presentamos la metodología utilizada para el análisis exploratorio de las instituciones de educación superior estudiadas, con el detalle de los programas de educación en alternancia que presentan junto a una matriz comparativa (en el anexo) de cada institución en el momento presente. Seguidamente la discusión de los resultados y las conclusiones, nos permiten identificar los elementos clave de un modelo transfronterizo así como un conjunto de acciones que contribuyen al desarrollo y la mejora de la empleabilidad necesaria en el territorio analizado.

\section{Revisión de la literatura}

La interacción de universidades/alumnos, empresas y gobiernos favorece el crecimiento y el empleo en zonas con nuevas visiones estratégicas (Etzkowitz, 2010; Bagherimoghadam et al. 2012; Ranga \& Etzkowitz 2013; Bektas \& Tayauova, 2014; Betz et al., 2016; Sarpong et al. 2015), en concreto en la literatura académica se ha tratado extensamente el modelo de la TH, que arranca de Leydesdorff y Etzkowitz, y se evidencian experiencias de diversos modelos de innovación basados en la TH (Cai \& Liu 2015; Su \& Hung, 2009). El concepto de TH también se ha utilizado como estrategia operacional para 
el desarrollo regional de zonas que quieren innovar, favorecer el desarrollo económico, optimizar los recursos, conseguir bienestar social (Hincapié et al. 2015) y promover la economía basada en el conocimiento como en Suecia (Jacob 2006), Etiopía (Saad et al., 2008), Brasil (Almeida 2005). Además, en la actual revolución tecnológica, a través de los contratos Universidad-Empresa se activa un efectivo mecanismo como nexo necesario entre la universidad y el sector productivo, para la generación del desarrollo científico-técnico y económico (Castillo \& Reyes, 2015).

Diferentes autores avalan la incidencia de la $\mathrm{TH}$ en la política y desarrollo de los territorios (Inzelt, 2004; Marques et al. 2006; Cooke y Leydesdorff 2006; Saad et al. 2008; Brännback et al. 2008; Dezhina, 2014; Doh y Kim, 2014; Balzer y Askonas 2016), por ello, para potenciar la transformación competitiva del territorio transfronterizo entre actores de ambos lados de los Pirineos se debe tener presente la TH.

Es importante tener presente que el modelo evolutivo de la TH se enmarca en un contexto de cambios económicos globales que inciden con diferente intensidad en ámbitos geográficos y por tanto varía entre regiones y países, de manera que el ámbito geográfico que nos ocupa (Euskadi, Sur de Francia y Cataluña) puede presentar diferentes intensidades (LawtonSmith \& Leydesdorff, 2014) que se deben gestionar correctamente.

Cabe tener presente que el Espacio Europeo de Educación Superior ha propiciado un mayor acercamiento entre la enseñanza superior y el mundo laboral, para otorgar empleabilidad a la población activa. Además, autores como Van der Hofstadt y Gómez (2006) señalan que el estudiante debe aprender las competencias genéricas que le asistan para conseguir y mantener un empleo y otros abogan por un aprendizaje activo propiciando el desarrollo del pensamiento crítico que es, frente a otras enseñanzas superiores enfocadas a la profesionalización, el valor añadido de la enseñanza universitaria. Todo con voluntad de formar a personas con las competencias y habilidades necesarias para el desarrollo profesional. La adquisición de competencias en educación superior, a partir de la alternancia, se traduce en cambios profundos en la docencia, las metodologías, los modelos formativos, considerando la alternancia como un nexo de unión entre teoría y práctica (Coiduras, 2013). Además, las competencias profesionales se adquieren mejor en un contexto real, con situaciones complejas y con acceso a medios profesionales. Esta situación nos remite ineludiblemente a los escenarios socioprofesionales, en la medida que dichos escenarios son genuinos para la adquisición y desarrollo de competencias profesionales. La mediación experta en la universidad y en el ámbito profesional han de facilitar esta integración para superar la simple observación y acción y tomar conciencia sobre la propia movilización de recursos y competencias 
en la actuación profesional (Coiduras et al., 2015). Por lo tanto, desarrollar un sistema de educación en alternancia efectivo, requiere de cierto grado de responsabilidad y reflexión sistémica para que realmente la situación de trabajo se convierta en situación de aprendizaje. La clave reside en la simbiosis conocimiento y escenario profesional como mejor vía para la inserción laboral de los estudiantes universitarios (Pereira \& Solé, 2013).

La formación en el contexto de trabajo, argumenta Levy-Leboyer (1997), es superior a cualquier tipo de formación, por cuanto «las experiencias obtenidas de la acción, de la asunción de responsabilidad real y del enfrentamiento a problemas concretos, aportan realmente competencias que la mejor enseñanza jamás será capaz de proporcionar». Además, el aprendizaje en un ámbito productivo real es clave para una formación integral y la formación en alternancia se consolida como un puente entre el sistema productivo y los centros educativos, en sintonía con las demandas reales del contexto presente, como mecanismo para la oportuna inserción laboral (Sarceda \& Rial, 2011).

Actualmente en esta zona transfronteriza existen experiencias que favorecen la interacción entre universidad/alumnos-empresas-gobiernos en aras de conseguir una zona transfronteriza que innove y cree empleo para sus habitantes. Cabe resaltar que actualmente la TH debe contemplar la sostenibilidad en sintonía con el cambio derivado de las nuevas relaciones socioeconómicas (Nicolaevich et al. 2015), y la innovación y el espíritu empresarial deben entenderse como el resultado de la interacción de numerosos agentes económicos (Ferrary \& Granovetter, 2009).

\section{Metodología exploratoria: planteamiento cualitativo, descriptivo y comparativo}

El estudio exploratorio se realiza en instituciones de educación superior, a ambos lados de la frontera entre Francia y España. Los criterios de selección de las cuatro instituciones objeto de análisis derivan de sus características comunes en cuanto a su tamaño, sus ámbitos de conocimiento y su entorno empresarial, por lo que dichas instituciones son socias de un proyecto competitivo de innovación educativa. Las instituciones objeto de estudio son: la Universidad de Mondragón, la Universidad de Vic-Universidad Central de Catalunya (en España), la École Superieure des Technologies Industrielles Avancées (ESTIA) y la Université de Pau (en Francia) y presentan experiencias en sintonía con el modelo TH por la interacción entre alumnos, empresas y gobiernos, para incentivar la innovación educativa y el desarrollo territorial con una economía basada en el conocimiento. El análisis exploratorio de los proyectos de estas 
instituciones, junto con el estudio descriptivo y comparativo de las mismas mediante diferentes variables como: el tipo de educación en alternancia que utilizan, la normativa que les ampara, el tipo de estudios (doctorado, máster, grado) en alternancia, las competencias y los resultados de aprendizaje conseguidos, permite desarrollar la metodología de estudio. De otra parte, el trabajo de campo ha consistido en diversas visitas y reuniones entre los cuatro socios del proyecto, en cada uno de los territorios geográficos, para poner en común sus memorias y planes de estudios oficiales y la educación superior en alternancia de cada institución, con voluntad de acercar posiciones e interaccionar con transversalidad. En el anexo 1 se incluye una matriz comparativa de los 4 modelos transfronterizos de alternancia.

\section{Formaciones en alternancia en los Grados en alternancia en la Universidad de Mondragón}

En el curso 2014-2015 se implantó en Enpresagintza, la Facultad de Empresariales de la Universidad de Mondragón, una fase piloto de un Grado en Alternancia en Administración y Dirección de Empresas. Mondragón Unibertsitatea es una Universidad Cooperativa perteneciente al Grupo Mondragón y se caracteriza por su capacidad innovadora y su cercanía al tejido empresarial, dando respuesta a las necesidades que demanda el mercado. De hecho, Mondragón Unibertsatea fue pionera en el desarrollo del modelo educativo basado en la adquisición de competencias, el cual ha sido validado y mejorado con adecuación a los estudios universitarios al Espacio Europeo de Educación Superior (Bolonia). Este desarrollo ha posibilitado que actualmente oferte un Grado en Administración y Dirección de Empresas en Alternancia que, internamente, se conoce como el proyecto myGade. Este Grado está reconocido por Aneca. El modelo myGade se basa en el concepto de Work based learning y tiene como objetivo fundamental mejorar la capacitación de los jóvenes para incorporarse al mercado laboral, incidiendo en la mejora de los conocimientos y habilidades requeridas (competencias), además de los valores y las actitudes. De hecho, se considera que estas últimas poseen un efecto multiplicador en el proceso de aprendizaje y que solo pueden trabajarse a través de una experiencia real en las empresas. Para ello, se ha creado un ecosistema de aprendizaje en el que no solo participan la universidad y el alumnado, sino que la organización se incorpora como tercer agente protagonista en este proceso. Esto supone beneficios contrastables para todas las partes, dado que la empresa es el contexto real más adecuado para adquirir ciertas competencias, habilidades y actitudes.

Durante los estudios el alumnado debe adquirir una serie de competencias técnicas, trasversales y profesionales definidas. La adquisición de las competencias técnicas, principalmente, se realiza en la aulas de la 
universidad, mientras que la experiencia en empresas reales permite al alumno adquirir las competencias transversales (ej. competencias digitales, comunicación efectiva, competencias informacionales, pensamiento orientado a la resolución de problemas, trabajo en equipo, visión global del equipo y aprender a aprender) y profesionales (adaptabilidad al entorno, iniciativa y proactividad, autoconfianza, responsabilidad). La formación en alternancia se concibe como una propuesta de aprendizaje/enseñanza que transforma situaciones de trabajo en situaciones de aprendizaje, generando un proceso de formación flexible y permanente a partir de la capacidad para aprender en y para el trabajo, y para detectar, por parte de los sujetos participantes, sus necesidades de formación. Esta propuesta posibilita que las personas en su vivencia individual integren los tiempos y espacios de su formación y de su trabajo, alternando y aplicando los componentes formativos (descripción, sistematización, síntesis, reflexión, expresión oral y escrita, entre otros) a la situación, organización y contenido y/o naturaleza del trabajo. La alternancia es un proceso que contribuye a la construcción del aprendizaje desde el hacer, pero que a su vez requiere de la conciencia de la persona respecto a lo aprendido, así como de los conocimientos teóricos requeridos para generar el saber hacer en sintonía con la TH. Así, siguiendo a Kolb (1984) se puede hablar de la alternancia como un aprendizaje experiencial clave en torno a la cual se organiza el desarrollo personal, en función del trabajo y de la educación de las personas. Se trata de una estrategia dinámica, que se construye y reconstruye en relación con la realidad para mantener en permanente relación el conocimiento teórico y el práctico entre universidades, empresas y gobiernos como prescribe la TH.

\section{Formaciones en alternancia en los Doctorados Industriales en Catalunya}

Un proyecto en este ámbito que presenta la zona de Catalunya en convergencia con un potencial modelo replicable en la zona objeto de estudio es: el Doctorado Industrial. El Doctorado Industrial (DI) es una clara respuesta al reto de la TH. El Plan de Doctorados Industriales es una estrategia del gobierno de la Generalitat de Catalunya, en colaboración con universidades públicas y privadas, con el objetivo de contribuir a una mayor competitividad e internacionalización del tejido industrial catalán, a la retención de talento y al fomento de doctorandos desarrollando proyectos de $\mathrm{I}+\mathrm{D}+\mathrm{i}$ en una empresa. Los agentes implicados en el Plan de Doctorados Industriales son: la empresa, la Universidad, el estudiante de doctorado y el Gobierno de la Generalitat de Catalunya (elementos propios de la TH). Esta es una iniciativa de la Secretaría de Universidades e Investigación gestionada por AGAUR (Agència de Gestió d'Ajuts Universitaris i de Recerca) y contempla empresas de diferentes actividades económicas y tamaños. El Doctorado Industrial identifica la interacción entre Generalitat de Catalunya- 
Universidad-Empresa, en concreto, se trata de un proyecto de investigación estratégico de una empresa, donde el doctorando desarrolla su formación investigadora, en colaboración con la Universidad, y es objeto de una tesis doctoral. La Generalitat de Catalunya da apoyo económico a estos proyectos con cofinanciamiento o con ayuda específica y anualmente establece un número máximo de proyectos a subvencionar de cada modalidad. La tesis doctoral se desarrolla en el marco de un convenio de colaboración entre la universidad o centro de investigación, la empresa y el doctorando con el apoyo económico de la Generalitat de Catalunya.

El doctorando, con una nota mínima para su acceso al programa, dispone de un director de tesis vinculado a la Universidad o centro de investigación y un responsable de la empresa (ubicada en Catalunya) y distribuye su dedicación entre la universidad y la empresa. De otra parte, el doctorando participa en programas formativos en competencias específicas relacionadas con el liderazgo, la coordinación y la gestión de proyectos $\mathrm{I}+\mathrm{D}+\mathrm{i}$, la transferencia de resultados de investigación, el desarrollo de nuevas empresas y la propiedad intelectual e industrial, entre otras materias relevantes, y dispone de una asignación anual (bolsa de movilidad), financiada por la Generalitat de Catalunya, cuya finalidad es la asistencia a seminarios y congresos relacionados con el proyecto de investigación, así como estancias en otras sedes de la empresa, en otras universidades o en centros de investigación de fuera de Catalunya, con el límite temporal de los tres años de duración del Doctorado Industrial.

El director de tesis debe formar parte de un grupo de investigación reconocido (SGR) por la Generalitat de Catalunya o bien ser investigador del programa ICREA o haber obtenido financiación del Consejo Europeo de Investigación (ERC). De otra parte, todas las tesis leídas del Doctorado Industrial tienen la mención de doctorado industrial y empresas y directores académicos involucrados reciben un reconocimiento de participación. Esta formación supone diversos casos de éxito que se pueden consultar en la web de la Generalitat de Catalunya (http://doctoratsindustrials.gencat.cat/ es) con reconocidos avales de calidad (Banal-Estañol et al. 2013; Salimi et al. 2015). A las empresas les permite atraer a personas con conocimientos y competencias de alto valor añadido, acceder a los grupos más punteros de universidades y centros de investigación y a sus equipamientos e infraestructuras, así como obtener ayudas económicas y beneficiarse de las deducciones y bonificaciones fiscales de las actividades de $\mathrm{I}+\mathrm{D}+\mathrm{i}$.

Para las universidades y los centros de investigación de Catalunya (CERCA), los proyectos de doctorado industrial son una ocasión para transferir su tecnología y sus conocimientos al entorno productivo y fortalecer los vínculos con el mundo empresarial. 
Para los estudiantes, constituye una magnífica oportunidad para incorporarse a un entorno innovador que combina la supervisión académica de la tesis con la tutoría de las empresas en el marco de un contrato laboral de tres años de duración y que incorpora, además, otras ventajas como la matrícula gratuita o una bolsa de movilidad. El modelo del doctorado industrial integra los elementos clave de la $\mathrm{TH}$.

Hay iniciativas en diferentes países europeos para incrementar sinergias entre universidades y empresas (Bélgica, Holanda, Alemania, Francia y Dinamarca) en concreto la Comisión Europea contempla el programa European Industrial Doctorates, para unir el mundo académico y el industrial con importantes becas de financiación en concordancia con la TH y existe literatura que avala la financiación en este ámbito (Benner \& Sandström, 2000). Estos programas científicos de la Comisión Europea, prescriben que la industria y la universidad tienen que pertenecer a países diferentes, por los beneficios que reportan a la empresa, la universidad y la sociedad en general.

De otra parte, la cooperación industria y doctorado industrial en la literatura académica refleja la consecución de más patentes para las industrias y más producción científica para las universidades (Salimi et al. 2015) en sintonía con la economía del conocimiento. También es destacable el acceso más rápido al mundo laboral una vez finalizado el doctorado industrial tal como avala Clarisse Angelier, directora de Formation par la Recherche de la Association Nationale de la Recherche et de la Technologie de Francia y la empresa Novo Nordisk de Dinamarca (que indica que los doctorados industriales favorecen el registro de patentes, el empleo y la mejora de beneficios brutos; añadiendo que en un 50\% son contratados posteriormente). Para el caso concreto de Catalunya, coordinadores del programa de Doctorados Industriales en grupos empresariales, como Agbar, destacan la experiencia positiva de acercar universidad y empresa, con el apoyo económico de la Generalitat de Catalunya.

A nivel de las declaraciones de los doctorandos la mayoría indica que el programa de doctorados industriales, es de gran acierto, ya que es un programa dual que permite a la vez obtener el título de doctor y ganar experiencia en el mundo laboral. De manera que facilita la empleabilidad y la investigación académica (tal como indica Clarisse Angelier).

De este programa, cuyos elementos son los de la TH, es destacable, el fomento a la movilidad internacional. Un aspecto delicado del DI es la duración limitada a 3 años, cosa que supone una gran coordinación entre universidad/empresa y gobierno, y en el caso de tesis por compendio de 
publicaciones académicas indizadas y de impacto en ese período de realización de tesis con financiación es muy ajustado.

Finalmente cabe tener presente que para el gobierno catalán los doctorados industriales son un importante objetivo para transferir el conocimiento recíprocamente del mundo académico al tejido empresarial (tal como dispone el director general de Universidades).

Formaciones en alternancia en la École Superieure des Technologies Industrielles Avancées (ESTIA) (Francia)

ESTIA se identifica como una Escuela de Educación Superior de Tecnologías Industrailes Avanzadas (ESTIA) que acoge a 700 ingenieros anualmente, transfiere tecnología y ayuda a la creación de empresas tecnológicas con especialidades en diseño de productos, electrónica, automática y organización y gestión industrial. Su campus está formado por un ecosistema de estudios de ingeniería y másteres; equipos de investigación y plataformas técnicas y una incubadora de empresas. Los alumnosingenieros mediante un aprendizaje en alternancia realizan prácticas con estrecha colaboración con las empresas de la zona, colaborando activamente con clústeres y polos de competitividad, aunque ESTIA dispone de una amplia red de empresas y colaboradores a nivel internacional. ESTIA es una de las primeras escuelas en Francia que utiliza el aprendizaje en alternancia desde hace 25 años.

Los estudiantes de ingeniería alternan estancias en la escuela de ingeniería y la correspondiente empresa por períodos de tres meses, es decir, tres meses en ESTIA y tres meses en la empresa y un tercio de los estudiantes llevan a cabo la alternancia como aprendices asalariados con un contrato de trabajo siguiendo en paralelo la formación de ingeniero. Además, durante estos períodos los estudiantes aprendices puede realizar movilidad por toda Francia y evidentemente trabajar en su región de origen. El contrato entre ESTIA y la empresa, tiene en cuenta las necesidades de las empresas y de los estudiantes y es a tiempo completo. El contrato se firma por el responsable de la empresa, el estudiante-aprendiz y el centro de formación ESTIA, se trata de una formación oficial avalada por el gobierno, en sintonía con la TH, con las tasas de aprendizaje establecidas según normativa. La alternancia se puede iniciar en el primero o segundo año y el periodo de formación es el mismo en estudiantes con o sin alternancia. El Ingeniero ESTIA recibe una formación científica y tecnológica combinada con una fuerte cultura industrial, que le prepara para tres tipos de funciones; el diseño del producto, el control de los procesos automatizados y la organización y gestión industrial. Las ventajas para el estudiante son obtener el título 
E. TARRATS-PONS, N. ARIMANY-SERRAT, S. ARANDO-LASAGABASTER, M. MARTÍNEZ-GONZÁLEZ,

de ingeniero y una experiencia laboral al mismo tiempo que recibe una retribución salarial durante el periodo de formación.

Es relevante, tener presente que en Francia la regulación de la tasa de aprendizaje facilita la alternancia que lleva a cabo ESTIA.

\section{Formaciones en alternancia en la Université de Pau (Francia)}

En la Universidad de Pau, concretamente en la Escuela Universitaria de Management (IAE), se llevan a cabo 9 másteres en alternancia, que se desarrollan con verdadera interacción entre universidad y empresas; en concreto, el estudiante mientras desarrolla el máster realiza una semana de clases en el centro de educación superior y tres semanas de trabajo en la empresa consiguiendo una formación integral teórica y práctica. Además, la educación superior en alternancia está avalada jurídicamente, es decir, la ley de 5 de marzo de 2014 respalda este tipo de formación y la tasa de aprendizaje, regulada en Francia, provee la adecuada financiación para que se pueda desarrollar correctamente la alternancia entre universidad y empresa en la educación superior. De manera que es un modelo acorde con la TH ya que dispone de los mismos elementos de interacción: Universidad/ alumnos, empresas y gobierno que identifica el camino a seguir.

La Ley n. ${ }^{\circ}$ 2014-288, de 5 de marzo 2014, transpone el Acuerdo Nacional Interprofesional (ANI), de 14 de diciembre 2013, y realiza así una amplia reforma del sistema de formación profesional francés; además de implementar la Cuenta Personal de Formación (CPF), de manera que la ley francesa reordena varios de los principios que rigen la formación profesional y reconsidera el sistema de financiación, instituyendo una contribución única que debe abonar cada empresa a un organismo colector.

En la ley se refuerza el control de los fondos de aprendizaje mediante el artículo 34, de manera que existe mayor control de los medios puestos a disposición de las empresas para estas formaciones, y un mayor control de las informaciones oportunas de los actores que intervienen en el aprendizaje.

La tasa de aprendizaje, que consiste en un impuesto legal a través del cual las empresas participan en la financiación de la formación tecnológica, profesional y de aprendizaje, identifica la participación del gobierno como un elemento de la TH que interacciona entre universidades y empresas; esta tasa particular francesa se calcula sobre la base de la remuneración pagada y su pago se acompaña de una contribución adicional para el aprendizaje. 
Es decir, la tasa de aprendizaje se destina a financiar el desarrollo de la primera formación tecnológica, profesional y de aprendizaje.

Para el caso concreto de l'École Universitaire de Management IAE PauBayonne su tasa se fija en el 0,68\% de la nómina para el año natural 2015. De otra parte, en l'École Universitaire de Management IAE Pau-Bayonne la alternancia permite la constante interacción entre empresa, universidad, alumno y gobierno (en sintonía con la TH).

Para el alumno permite diseñar un proceso profesional completo gracias a las competencias teóricas de formación oficial de la universidad y las técnicas adquiridas y capacitación en el entorno laboral. Así pues, el alumno desarrolla las competencias prácticas en sintonía con las competencias teóricas adquiridas en la universidad para completar su formación según los parámetros de la alternancia, con los elementos propios de la TH. Cabe resaltar que esta formación en alternancia, facilita en gran manera que el alumno consiga empleo, integre en su persona la cultura empresarial y adquiera la autonomía necesaria para defender su vida laboral.

\section{Caracterización, discusión y resultados de la educación en alternancia en las cuatro instituciones de educación superior}

En las cuatro instituciones de educación superior la alternancia permite acercar posiciones entre las organizaciones educativas y el entorno empresarial, hasta el punto de que una de las instituciones educativas (Universidad de Mondragón) tiene la forma jurídica de cooperativa, forma jurídica propia del grupo empresarial Mondragón integrado por cooperativas autónomas. En el caso de Mondragón, al ser una cooperativa integrante del grupo Mondragón, favorece esa necesaria alternancia entre universidad y empresa con el objetivo de formar a los estudiantes como futuros actores del entorno laboral actual con las competencias y habilidades propias de nuestros días.

En el caso de los doctorados industriales de Catalunya, este modelo facilita la aproximación necesaria entre la academia y las empresas fomentando más patentes para las empresas y más investigación de prestigio por parte de las universidades del territorio.

En el territorio francés, los ingenieros ESTIA, mediante la alternancia, se acercan a la industria mediante la colaboración en proyectos industriales reales con formación científica y tecnológica basada en títulos oficiales combinados con la oportuna cultura industrial adquirida en las empresas, 
siguiendo la oportuna regulación de la tasa de aprendizaje establecida en Francia.

En la Universidad de Pau los diferentes másteres en alternancia permiten conseguir la deseada empleabilidad de la zona mediante la integración de la academia y la cultura empresarial, y adquirir la autonomía necesaria para defender un empleo ya que el alumno de máster oficial desarrolla las competencias prácticas en sintonía con las competencias teóricas adquiridas en la universidad para completar su formación según los parámetros de la alternancia, con los elementos propios de la TH.

Las cuatro experiencias integran los elementos propios de la Triple Hélice (TH) es decir los elementos que caracterizan la alternancia resultan de la interacción entre las instituciones de educación superior, los alumnos, las empresas y los gobiernos de las cuatro zonas, ya que se trata de títulos oficiales para cada país que recogen en sus planes de estudio la alternancia como modelo de innovación educativa que facilita la incorporación al mercado laboral de los alumnos de educación superior en cada zona. Asimismo, al colaborar las cuatro instituciones de educación superior en un proyecto de innovación educativa, por su similitud en tamaño, ámbitos de conocimiento y entorno empresarial, se pretende la interacción de las mismas de manera transversal para potenciar la alternancia y el desarrollo de las zonas especialmente en empleabilidad y economía basada en el conocimiento. La interacción en el proyecto se traduce a nivel académico en codirección de tesis, artículos académicos conjuntos en el ámbito de la innovación educativa y convenios de trasferencia colaborativa e investigación entre las cuatro zonas. En el ámbito empresarial se plantea como futuro trabajo la creación de un Observatorio de las empresas de las cuatro zonas para facilitar la transversalidad y los retos estratégicos con la oportuna comunicación y visibilidad web a través de un portal de investigación conjunta y transferencia colaborativa.

\section{CONCLUSIONES}

En este estudio exploratorio identificamos 4 prácticas de alternancia e innovación educativa en la zona transfronteriza (dos experiencias en España y dos en Francia) susceptibles de ser aplicadas con transversalidad en la zona objeto de estudio.

Según la metodología utilizada, el planteamiento descriptivo y comparativo de las cuatro experiencias de educación en alternancia permite identificar un análisis integrado con aspectos comunes y discrepantes. 
Las evidencias del análisis presentado identifican como aspectos fundamentales:

1. Una clara correspondencia entre el modelo de la $\mathrm{TH}$ y las 4 experiencias de educación en alternancia presentadas, ya que las cuatro conciben la formación en alternancia como una inversión compartida entre universidad/alumnos, empresas y gobiernos tal como establece el modelo de la TH (Inzelt, 2004; Marques et al. 2006; Cooke y Leydesdorff 2006; Saad et al. 2008; Brännback et al. 2008; Dezhina, 2014; Doh y Kim, 2014; Balzer y Askonas 2016).

2. En las cuatro experiencias se evidencia una mayor empleabilidad con la educación en alternancia en sintonía con la competitividad que identifica el modelo de la TH (Etzkowitz, 2010; Bagherimoghadam et al. 2012; Ranga y Etzkowitz 2013; Bektas, 2014; Betz et al., 2015; Sarpong et al. 2015).

3. En las cuatro experiencias los títulos que amparan la educación en alternancia son oficiales, con normativas jurídicas regulatorias para cada caso (en las dos experiencias francesas existe una tasa impositiva reconocida en la ley francesa que apoya la educación en alternancia a diferencia de la normativa española). Además, son procesos educativos inductivos entre universidad y entorno laboral como en la TH (Coiduras, 2013).

4. En las cuatro zonas geográficas entre Francia y España la educación en alternancia es concebida como una innovación educativa tal como se concibe la TH (Inzelt, 2004; Marques et al. 2006; Cooke y Leydesdorff 2006; Saad et al. 2008; Brännback et al. 2008; Dezhina, 2014; Doh y Kim, 2014; Balzer y Askonas 2016). Dicha educación en alternancia en la Universidad de Pau y Universidad de Vic es para estudios de máster y doctorado y en la Universidad de Mondragón y Estia para los estudios de grado.

5. Las competencias vinculadas al modelo de alternancia, aunque presentan diferencias en las cuatro instituciones (anexo) permiten mejorar la transferencia de los conocimientos adquiridos y conseguir unos resultados de aprendizaje que facilitan la empleabilidad en cada zona.

6. La interacción de las cuatro zonas en un proyecto competitivo de innovación educativa permite cooperar a nivel académico (codirección de tesis, coautoría en publicaciones académicas, transferencia de conocimiento) y profesional (mediante la creación de un observatorio de los principales clústeres empresariales de las zonas analizadas con la visibilidad web oportuna).

Como limitación al estudio son destacables las diferentes normativas entre los dos países que complican la posibilidad de un modelo único de 
educación en alternancia para las cuatro zonas analizadas entre Francia y España.

La prospectiva de investigación consistirá en valorar y validar si la codirección de tesis, la coautoría de artículos, así como las prácticas empresariales entre los alumnos de las diferentes instituciones de educación superior analizadas y las empresas de estas zonas, permiten junto con la transferencia de conocimiento oportuna un organigrama de un modelo en alternancia transfronterizo, replicable a otras zonas.

\section{NOTAS}

1 Este dato corresponde a Euroarea (18 países). 


\section{REFERENCIAS BIBLIOGRÁFICAS}

Abdala, E. (2004). Formación por alternancia. Un esbozo de la experiencia internacional. En E. Abdala, J. Díaz Zamúz, J. Lasidam, S. Latorre, S. Santos \& M. Suanes. Formación de jóvenes en alternancia. Una propuesta pedagógica innovadora (pp. 11-28). Montevideo: CINTERFOR/ OIT, CEPAP, El Abrojo.

Almeida, M. (2005). The evolution of the incubator movement in Brazil. International Journal of Technology and Globalisation, 1(2), 258-277.

Bagherimoghadam, N., Hosseini, SH. \& SahafZadeh, M. (2012). An analysis of the industry-government-university relationships in Iran's power sector: A benchmarking approach. Technology in Society, 34(4), 284-294.

Balzer, H. \& Askonas, J. (2016). The Triple Helix after communism: Russia and China compared. Triple Helix 3(1). 10.1186/s40604-015-0031-4

Barnett, R. (1993). Knowledge, Higher Education and Society: a Postmodern Problem. Oxford Review of Education, 19(1), 33-46.

Bekta囚, Ç. \& Tayauova, G. (2014). A Model Suggestion for Improving the Efficiency of Higher Education: University-Industry Cooperation. Procedia - Social and Behavioral Sciences, 116, 2270-2274.

Benner, M. \& Sandström, U. (2000). Institutionalizing the triple helix: research funding and norms in the academic system. Research Policy, 29 (2000), 291-301.

Betz, F., Carayannis, E., Jetter, A., Min, W., Phillips F. \& Shin, DW. (2016). Modeling an Innovation Intermediary
System Within a Helix. Journal of the Knowledge Economy, 7(2), 587-599.

Banal-Estañol, A. \& Macho-Stadler, I. \& Pérez-Castrillo, D. (2013). Research output from university-industry collaborative projects. Economic Development Quarterly, 27 (1), 71-81.

Brännback, M. \& Carsrud, A. \& Kruege, N. \& Elfving, J. (2008). Challenging the triple hélix model of regional innovation systems: A venturecentric model. International Journal Technoentrepreneurship, 1(3), 257-278.

Cai, Y. \& Liu, C. (2015). The roles of universities in fostering knowledgeintensive clusters in Chinese regional innovation systems. Science and Public Policy, 42(1), 15-29. 10.1093/scipol/ scu018

Castillo, L. y Reyes, S. (2015). Los modelos de relación universidad-empresa. Revista Caribeña de Ciencias Sociales. Recuperado de https://goo.gl/xLGJ9Y

Coiduras, JL. (2013). Universidad y empleo: escenarios complementarios en educación superior. Formación XXI. Revista de Formación y Empleo, 22. Monográfico.

Coiduras, J., Isus, S. y Del Arco, I. (2015). Formación inicial de docentes en alternancia. Análisis desde las percepciones de los actores en una experiencia de integración de aprendizajes. Educar, 51(2), 277-297.

Comisión Europea (2012). Un nuevo concepto de educación: invertir en las competencias para lograr mejores resultados socioeconómicos. Estrasburgo: Comisión Europea. Recuperado de https://goo.gl/JfdSWH

Cooke, P. \& Leydesdorff, L. (2006). Regional Development in the 
Knowledge-Based Economy: The Construction of Advantages. Journal of Technology Transfer, 31(1), 5-15.

De Miguel, M. (2006). Metodologías de enseñanza y aprendizaje para el desarrollo de competencias. Orientaciones para el profesorado universitario ante el Espacio Europeo de Educación Superior. Madrid: Alianza

Dezhina, I.G. (2014). Technology platforms in Russia: a catalyst for connecting government, science, and business? Triple Helix, 1(1), 1-10.

Doh, S. \& Kim, B. (2014). Government support for SME innovations in the regional industries: The case of government financial support program in South Korea. Research Policy, 43(9), 1557-1569.

Etzkowitz, H. (2010). The Triple Helix. University-Industry-Government. Innovation in action. Monograph. Tomsk.

Eurostat (2014): Base de datos de estadísticas regionals. Recuperado de https://goo.gl/SD2z9s

Ferrary, M. \& Granovetter, M. (2009). The role of venture capital firms in Silicom Valley's complex innovation network. Economy and Society, 38(2), 326-359.

Hincapié, M., Moreno, G., Prada, A., Valencia, J. y Arango, D. (2015): Panorama de la innovación en el modelo de Triple Hélice: Universidad-EmpresaEstado. Espacios, 36 (24), 22-42.

Huerta Amezola, J.J., Pérez García, I.S. y Castellanos Castellanos, A.R. (2000). Desarrollo curricular por competencias profesionales integrales. Revista Educar, 87-96.

Inzelt, A. (2004). The evolution of university-industry-government relationships during transition. Research Policy, 33(6), 975-995.

Jacob, M. (2006). Utilization of social science knowledge in science policy: Systems of Innovation, Triple Helix and VINNOVA. Social Science Information, 45(3), 431-462.

Kolb, D.A. (1984). Experiential learning: Experience as the source of learning and development. New Jersey: Prentice-Hall.

Lawton-Smith, H. \& Leydesdorff, L. (2014). The Triple Helix in the context of global change: dynamics and challenges. Prometheus, 32 (4), 321-336.

Levi-Leboyer, C. (1997). La gestión de las competencias. Barcelona: Ediciones Gestión 2000.

Luzón Trujillo, A., Sevilla, D. y Torres, M. (2009). El Proceso de Bolonia: significado, objetivos y controversias. Revista de la Asociación de Inspectores de Educación en España, 10, 1-11.

Marques, JPC., Caraça, JMG. \& Diz, H. (2006). How can university-industrygovernment interactions change the innovation scenario in Portugal?the case of the University of Coimbra. Technovation, 26(4), 534-542.

Mourshed, M \& Farrell, D. \& Barton, D. (2016). Education to Employment: Designing a system that works. Recuperado de https://goo.gl/mmoGqD

Pereira, C. y Solé, J. (2013). La cualificación profesional en educación social. El papel de prácticum. Pedagogía Social. Revista Interuniversitaria, 21, 237-258.

Ranga, M. y Etzkowitz, H. (2013). Triple Helix systems: an analytical framework for innovation policy and practice in the Knowledge Society. Industry and Higher Education, 27(4), 237-262. 
Saad, M., Zawdie, G. \& Malairaja, C. (2008). The triple helix strategy for universities in developing countries: the experiences in Malaysia and Algeria. Science and Public Policy, 35(6), 431-443.

Salimi, N., Bekkers R. \& Frenken, K. (2015). Does working with industry come at a price? A study of doctoral candidates' performance in collaborativa vs. non-collaborativa $\mathrm{PhD}$ projects. Technovation, 41(1), 51-56.

Sarceda, M.C. y Rial, A. (2011). De las prácticas en alternancia a la inserción laboral: resultados de una investigación. Revista de Docencia Universitaria, 9(2), 231-252.

Sarpong, D. \& Abdrazak, A. \& Alexander, E. \& Meissner, D. (2015). Organizing practices of university, industry and government that facilitate (or impede) the transition to a hybrid triple helix model of innovation. Technological Forecasting and Social Change.

Su, Y. \& Hung, L. (2009). Spontaneous vs. policy-driven: The origin and evolution of the biotechnology cluster. Technological Forecasting and Social Change, 76(5), 608-619.

Van der Hofstadt, C. \& Gómez, J.M. (2006). Competencias y habilidades profesionales para universitarios. Madrid: Díaz de Santos.

World Economic Forum (2016). The Future of Jobs Employment, Skills and Workforce Strategy for the Fourth Industrial Revolution. Recuperado de https://goo.gl/yhKr9j 
E. TARRATS-PONS, N. ARIMANY-SERRAT, S. ARANDO-LASAGABASTER, M. MARTÍNEZ-GONZÁLEZ,

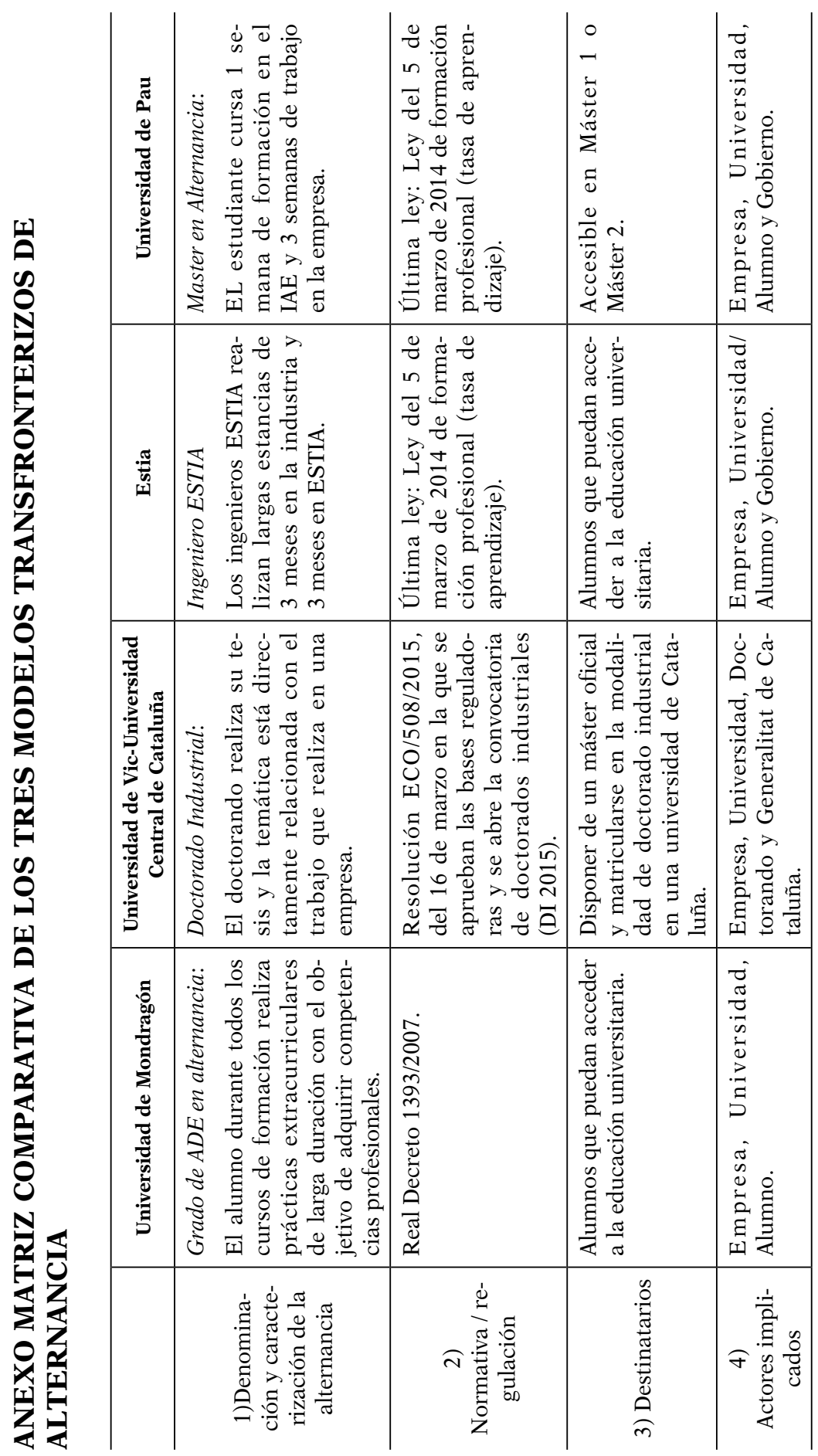


E. TARRATS-PONS, N. ARIMANY-SERRAT, S. ARANDO-LASAGABASTER, M. MARTÍNEZ-GONZÁLEZ, L. MATHIEU, M. SAMONNEAU, V. PILNIERI

EDUCACIÓN SUPERIOR EN ALTERNANCIA TRANSFRONTERIZA ENTRE FRANCIA Y ESPAÑA

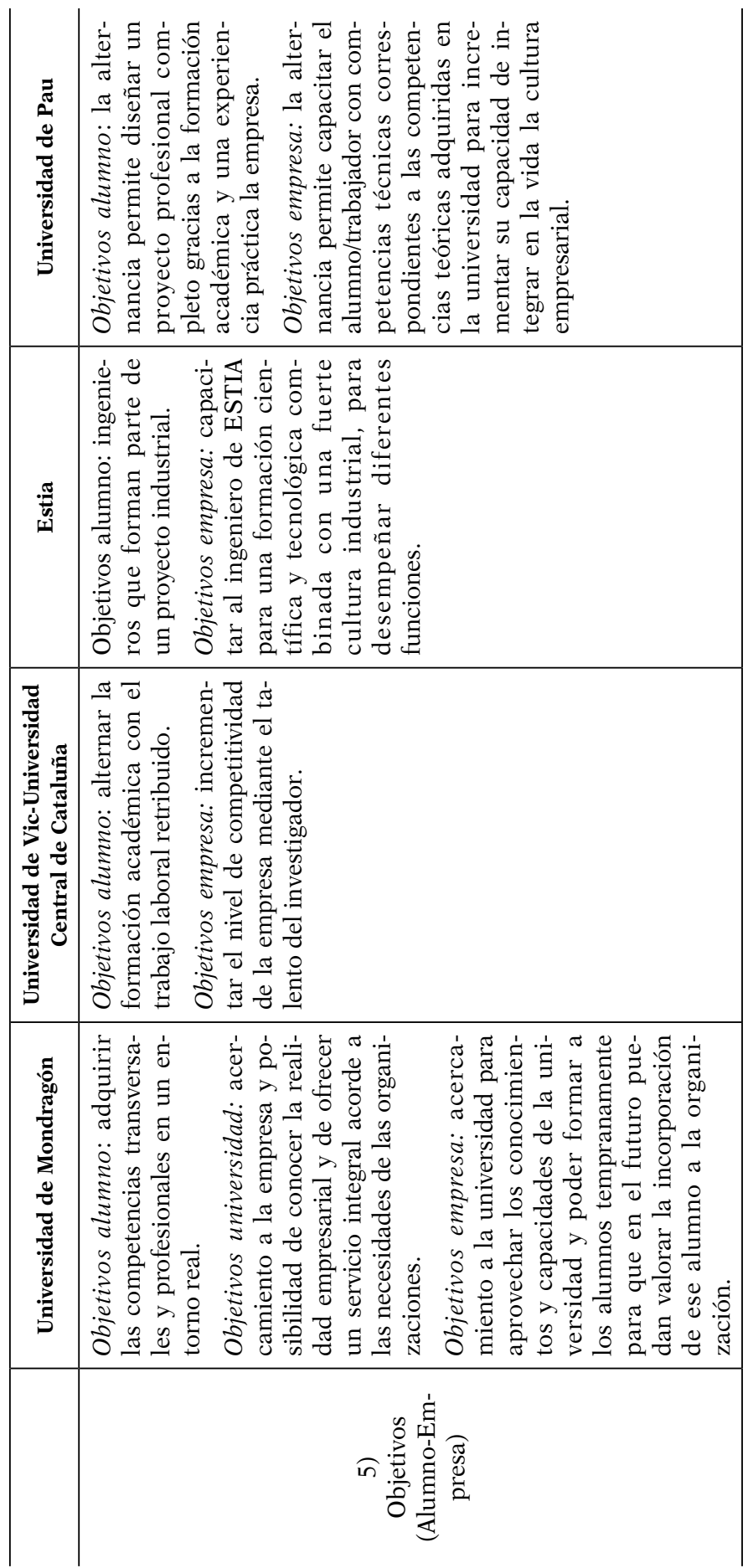


E. TARRATS-PONS, N. ARIMANY-SERRAT, S. ARANDO-LASAGABASTER, M. MARTÍNEZ-GONZÁLEZ,

EDUCACIÓN SUPERIOR EN ALTERNANCIA TRANSFRONTERIZA ENTRE FRANCIA Y ESPAÑA

\begin{tabular}{|c|c|c|c|}
\hline 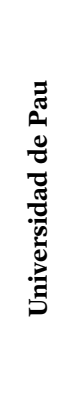 & 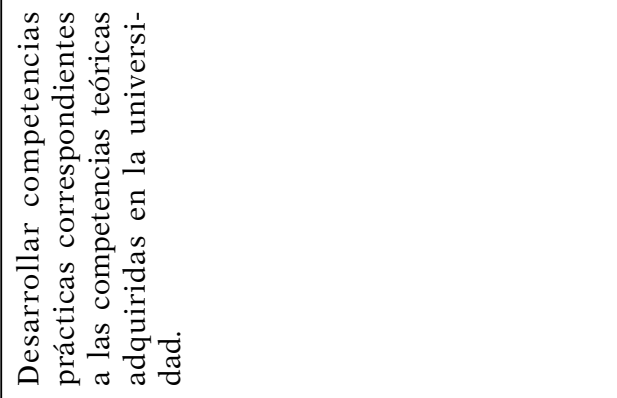 & 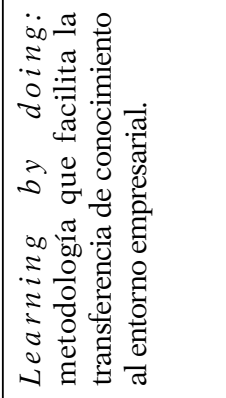 & 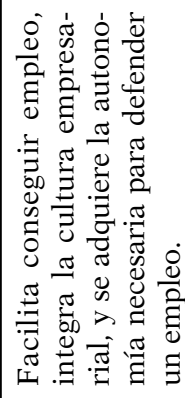 \\
\hline 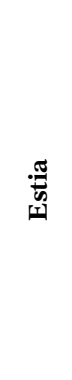 & 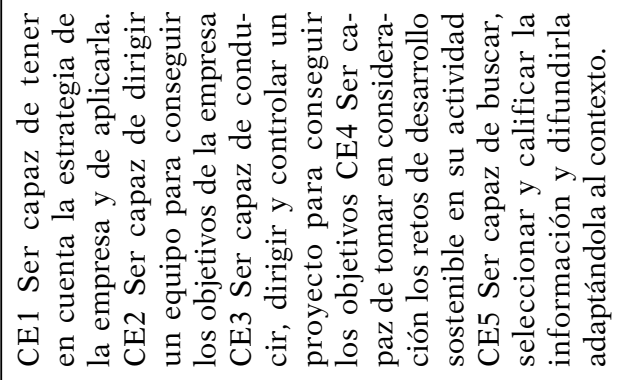 & 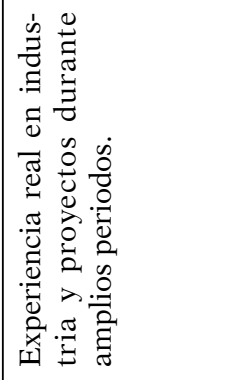 & 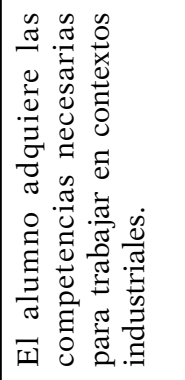 \\
\hline 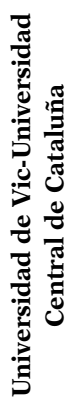 & 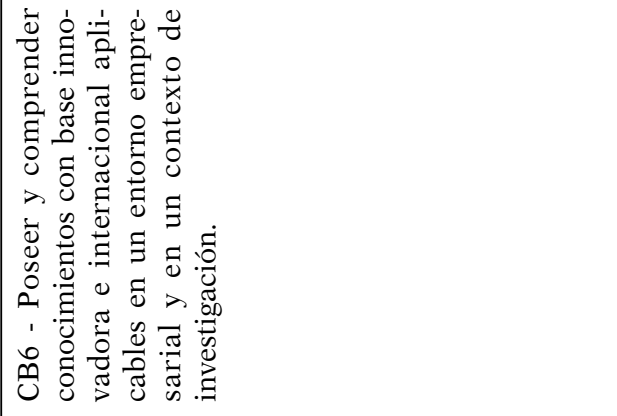 & 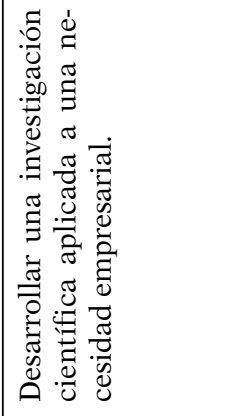 & 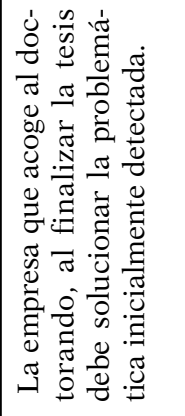 \\
\hline 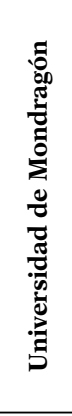 & 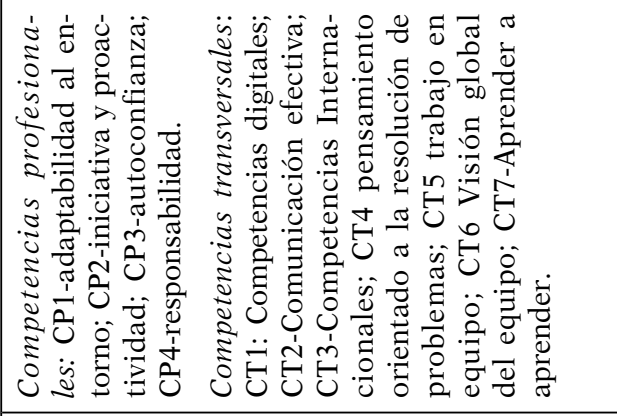 & 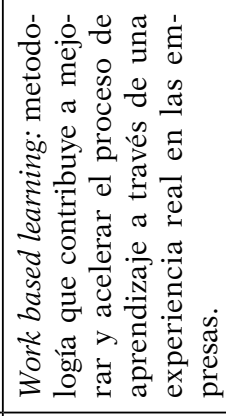 & 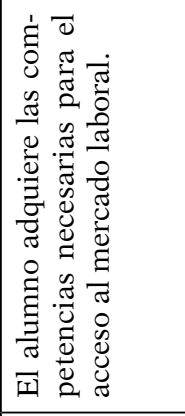 \\
\hline & 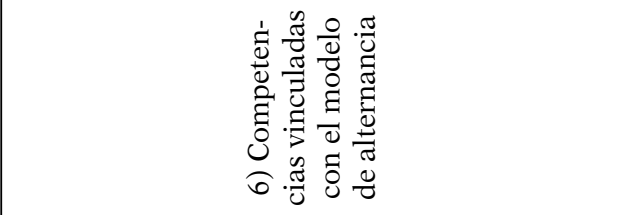 & $\widehat{\overbrace{}} \frac{\frac{\pi}{0}}{\frac{0}{0}}$ & 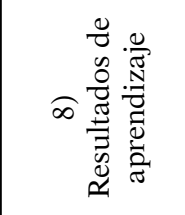 \\
\hline
\end{tabular}




\section{PERFIL ACADÉMICO Y PROFESIONAL DE LAS AUTORAS}

Elisenda Tarrats-Pons. Profesora agregada de la Universidad de Vic- Universitat Central de Catalunya. Directora del departamento de Economía y Empresa y coordinadora de la Unidad de Emprendimiento de la Universitat de Vic-Universitat Central de Catalunya. Ha participado en diversos proyectos competitivos. Sus líneas principales de investigación son el emprendimiento y el proceso de enseñanza y aprendizaje en la universidad, con especial interés en los métodos activos de aprendizaje con ponencias en diferentes congresos y publicaciones académicas indizadas.

Núria Arimany-Serrat, profesora titular de la Universitat de VicUniversitat Central de Catalunya. Coordinadora de los programas de doctorado de la Facultad de Empresa y Comunicación y del grupo de investigación Emprèn de la Universitat de Vic-Universitat Central de Catalunya. Ha participado en diversos proyectos competitivos. Sus principales líneas de investigación son la Responsabilidad Social Corporativa, el Análisis económico financiero y la innovación empresarial en diferentes clústeres con ponencias en diversos congresos y publicaciones académicas indizadas en estas áreas.

Saioa Arando Lasagabaster. Profesora Investigadora de la Universidad de Mondragón. Coordinadora Científica de la Facultad de Empresariales de MU y Coordinadora del área de conocimiento «Personas en cooperación: liderazgo y ownership». Ha participado en diversos proyectos competitivos y sus artículos han sido publicados en journals referentes. Sus líneas principales de investigación son la participación de las personas trabajadoras en las organizaciones, el cooperativismo y la gestión del talento.

Mariangélica Martínez Chávez. Profesora-Investigadora de la Facultad de Empresariales de Mondragón Unibertsitatea. Responsable del área de conocimiento de Estrategias Avanzadas. Ha participado en diversos proyectos competitivos. Sus líneas principales de investigación son la gestión de procesos de innovación y su traslación al proceso de enseñanza y aprendizaje.

Laetitia Mathieu. Responsable de las relaciones Internacionales y de los programas de movilidad de IAE Pau-Bayonne. Ha participado en diversos proyectos competitivos. Sus líneas principales de trabajo son la movilidad internacional de los estudiantes y el diseño y desarrollo de programas de alternancia.

Marion Saumonneau, responsable de proyectos. Interviene en la definición, gestión de proyectos y competencias. Interviene asimismo en 
formaciones iniciales y continuas. Sus líneas principales de investigación son pedagogías activas de aprendizaje e innovación de didácticas para la educación.

Véronique Pilnière, Profesora en Ciencias de Gestión y HDR (Habilitada para Dirigir Investigaciones y tesis doctorales), interviene en los ámbitos del cambio organizativo, la gestión de competencias y la gestión de riesgos. Formada en ergonomía y en ciencias cognitivas, acompaña a las empresas en el desarrollo de sus resultados. Además, está especializada en el modelo centrado en las interacciones de Palo Alto con el cual trabaja el Instituto Gregory Bateson (IGB) de Lieja fundado por Jean-Jacques Wittezaele.

Dirección de las autoras: Elisenda Tarrats-Pons

Núria Arimany-Serrat

Facultad de Empresa y Comunicación

Universidad de Vic-Universidad Central de

Cataluña

Calle Sagrada Família, 7

08500 Vic (Barcelona)

E-mail: elisenda.tarrats@uvic.cat

nuria.arimany@uvic.cat

Saioa Arando-Lasagabaster

Mariangélica Martínez-González

Enpresagintza, Facultad de Empresariales

Mondragon Unibertsitatea

Ibarra Zelaia, 2

20560 Oñati (Gipuzkoa)

E-mail: sarando@mondragon.edu

mmartinezc@mondragon.edu

Laetitia Mathieu

IAE Pau-Bayonne École

Universitaire de Management

8 allées des Platanes, Campus de la Nive

64100 Bayonne - FRANCE

E-mail: laetitia.mathieu@univ-pau.fr 


\section{Marion Samonneau}

Veronique Pilniere

ESTIA-École Supérieure des Technologies

Industrielles Avancées

97, Allée Théodore Monod

64210-Bidart - FRANCE

E-mail: m.saumonneau@estia.fr

v.pilniere@estia.fr

Fecha Recepción del Artículo: 22. Diciembre. 2016

Fecha Modificación del Artículo: 28. Marzo. 2017

Fecha Aceptación del Artículo: 20. Junio. 2017

Fecha Revisión para Publicación: 22. Febrero. 2018 